\title{
A Japanese diet and 19-year mortality: National Integrated Project for Prospective Observation of Non-Communicable Diseases and its Trends in the Aged, 1980
}

\author{
Yasuyuki Nakamura ${ }^{1,2}$, Hirotsugu Ueshima ${ }^{2}$, Tomonori Okamura ${ }^{2}$, Takashi Kadowaki ${ }^{2}$, \\ Takehito Hayakawa ${ }^{3}$, Yoshikuni Kita ${ }^{2}$, Robert D. Abbott ${ }^{2,4}$, Akira Okayama ${ }^{5}$ and for National Integrated \\ Project for Prospective Observation of Non-Communicable Diseases and its Trends in the Aged, 1980 \\ Research Group \\ ${ }^{1}$ Cardiovascular Epidemiology, Kyoto Women's University, 35 Imakumano Kitahiyoshi-cho, Higashiyama-ku, Kyoto 605-8501, \\ Japan \\ ${ }^{2}$ Department of Health Science, Shiga University of Medical Science, Otsu City, Shiga, Japan \\ ${ }^{3}$ Department of Hygiene and Preventive Medicine, Fukushima Medical University, Fukushima, Japan \\ ${ }^{4}$ Department of Epidemiology and Biostatistics, University of Virginia School of Medicine, Charlottesville, VA, USA \\ ${ }^{5}$ Department of Preventive Cardiology, National Cardiovascular Center, Suita City, Japan
}

(Received 4 April 2008 - Revised 22 September 2008 - Accepted 24 September 2008 - First published online 20 November 2008)

Few studies have examined the association between Japanese diet and mortality outcomes. We analysed the relationship between a healthy Japanese diet and all-cause and cause-specific mortality using the database from the National Integrated Project for Prospective Observation of Non-Communicable Diseases and its Trends in the Aged, 1980. At baseline in 1980, data were collected on study participants aged $\geqq 30$ years from randomly selected areas in Japan. We defined a measure of a healthy reduced-salt Japanese diet based on seven components from FFQ. The total score ranged from 0 to 7 , with 0 being least healthy and 7 being most healthy. Participants were divided into approximate tertiles of dietary scores (0-2, 3 and 4-7 scores). After excluding participants with co-morbidities, we followed 9086 participants (44\% men) for 19 years. There were 1823 all-cause and 654 cardiovascular deaths during the follow-up. With the dietary score group $0-2$ serving as a reference, the Cox multivariate-adjusted hazard ratios for groups with scores 3 and $4-7$ were 0.92 (95\% CI $0.83,1.04)$ and 0.78 (95\% CI $0.70,0.88)$ for all-cause mortality (trend $P<0.0001$ ), and 0.91 (95\% CI $0.75,1 \cdot 10$ ) and 0.80 (95\% CI 0.66, 0.97) for cardiovascular mortality (trend $P=0.022$ ). Adherence to a healthy reduced-salt Japanese diet was associated with an approximate $20 \%$ lower rate of all-cause and cardiovascular mortality.

Dietary pattern: Japanese diet: Cohort studies: Mortality

Recent interest in dietary patterns has spawned several studies of the associations between dietary patterns and longevity $^{(1,2)}$. Japanese cuisine is based on combining staple foods, typically rice or noodles, with soup, and side dishes made from fish, meat, vegetable, tofu and the like, designed to add flavour to the staple food. These are typically flavoured with dashi stock, made with katsuobushi (dried skipjack tuna flakes), miso and soya sauce, and are usually low in fat and high in salt. Since Japan is an island nation, people eat much seafood. Meat eating has been relatively rare. The beneficial aspects of the traditional Japanese diet have been attributed to its low intake of SFA and a high intake of PUFA, especially from fish. Long-term benefits include lower mortality from $\mathrm{CHD}$ and from some cancers, which contribute at least in part to Japanese having the longest life expectancy in the world ${ }^{(3,4)}$. A drawback of the Japanese diet is its high intake of salt and its association with a higher incidence and mortality from stroke and gastric cancer ${ }^{(5-7)}$. Presumably, if the Japanese diet is modified to emphasise the intake of foods that are low in salt, Japanese longevity could be increased further.

In the present study, we studied the preference for Japanese or Western diets, and from these data and those based on the previous studies, we comprehensively extracted the beneficial components of the Japanese diet and derived a healthy Reduced-Salt Japanese Diet Score. We analysed the relationship between the diet score and all-cause and cause-specific mortality using the database of the National Integrated Project for Prospective Observation of Non-Communicable Diseases and its Trends in the Aged, 1980 (NIPPON DATA80). The database includes more than 10000 participants from randomly selected regions in Japan, who were followed for 19 years $^{(8-10)}$. 


\section{Methods \\ Participants}

The participants in this cohort were those in the 1980 National Survey on Circulatory Disorders ${ }^{(8)}$. A total of 10546 community-based participants aged 30 years and above in 300 randomly selected health districts throughout Japan participated in the survey, which consisted of history taking, physical examinations, blood tests and a self-administered questionnaire on lifestyle, including an essential nutritional survey by the food-frequency method. For the present study, the participants were followed up to 1999 (National Integrated Project for Prospective Observation of Non-Communicable Diseases and its Trends in the Aged, 1980, 1980-99). The overall population aged 30 years and above in the participating health districts was 13771 . The participation rate was $76.6 \%$ (10546 of 13771 ) before exclusion for reasons mentioned later.

We reviewed the residence records of all the study participants for their vital status. In the cases of deaths, the causes were examined. To clarify the cause of death, we used the National Vital Statistics records. The underlying cause of death was coded according to the ninth International Classification of Disease for the National Vital Statistics until the end of 1994 and according to the tenth International Classification of Disease from the beginning of 1995 . Deaths were confirmed in each district by computer matching of data from the National Vital Statistics records using the district, sex and dates of birth and death as key codes.

Participants were excluded from follow-up because of a past history of coronary disease, stroke or significant co-morbidities such as renal insufficiency ( $n$ 539), because of missing baseline data ( $n$ 51) or because of a loss to follow-up ( $n 870$ ). The latter group was excluded because of the absence of a permanent address that was required for linking to National Vital Statistics records. The final sample comprised 9086 participants (4018 men and 5068 women). There were no significant differences between participants who were lost to follow-up and those who were included in the present study in terms of several risk factor characteristics. Therefore, the potential bias regarding the 870 participants lost to followup is thought to be negligible. Permission to use the National Vital Statistics records was obtained from the Management and Coordination Agency, Government of Japan. Approval for the present study was obtained from the Institutional Review Board of Shiga University of Medical Science for ethical issues (no. 12-18, 2000).

\section{Biochemical and baseline examinations}

The baseline surveys were conducted at public health centres. Baseline blood pressures (BP) were measured by trained research nurses using a standard mercury sphygmomanometer on the right arm of seated participants after at least $5 \mathrm{~min}$ of rest. Hypertension was defined as systolic $\mathrm{BP} \geq 140 \mathrm{mmHg}$, diastolic $\mathrm{BP} \geq 90 \mathrm{mmHg}$, or when a participant was receiving medications for the treatment of high BP. Height and weight were measured in stocking feet and light clothing. BMI was calculated as weight $(\mathrm{kg})$ divided by the square of height $\left(\mathrm{m}^{2}\right)$.

A lifestyle survey was also carried out using a self-administered questionnaire that asked about the typical daily consumption of thirty-one food items, as shown in Appendix. Egg consumption was coded as $\geq 2 \mathrm{eggs} / \mathrm{d}$, about $1 \mathrm{egg} / \mathrm{d}$, about $1 \mathrm{egg} / 2 \mathrm{~d}$, about $1-2 \mathrm{eggs} /$ week and less than once per week. Fish, meat and tsukemono (preserved roots or leaves of seasonal vegetables, e.g. cucumbers and aubergine, which are consumed with rice at the end of a meal) intake was coded separately as $\geq 2$ times/d, about 1 time/d, about 1 time/ 2d, about 1-2 times/week and less than once per week. The participants were also asked whether they frequently consumed soup with noodles, whether they used low-salt soya sauce and what their preferred type of diet was (Japanese, Western or mixed; Q19 in Appendix). They were enquired about their alcohol drinking habit (never, past, occasional and daily drinkers). Reported information was confirmed by public health nurses through interviews with the study participants regarding food consumption, smoking, drinking habit and present and past medical histories.

Non-fasting blood samples were drawn and centrifuged within $60 \mathrm{~min}$ of collection and stored at $-70^{\circ} \mathrm{C}$ until analyses. Serum total cholesterol, albumin, uric acid and creatinine were analysed in a sequential auto-analyser (SMA12/ 60, Technicon, Tarrytown, NY, USA) at a single laboratory (Osaka Medical Center for Health Science and Promotion). This laboratory is a member of the Cholesterol Reference Method Laboratory Network ${ }^{(11)}$. Serum concentrations of glucose were measured by the cupric-neocuproine method ${ }^{(12)}$ Diabetes was determined by medical history or defined as a serum glucose concentration $\geq 2000 \mathrm{mg} / \mathrm{l}$.

\section{Statistical analysis and components of the Reduced-Salt Japanese Diet Score}

Statistical Analysis Systems statistical software package version 9.1 for Windows (SAS Institute, Cary, NC, USA) was used throughout the analyses. We examined the relationship between the type of preferred diet and the frequency of dietary components from the nutritional survey. Then, we defined seven components from the nutritional survey to measure a healthy reduced-salt Japanese diet. The components included egg intake $\leq 2 \mathrm{eggs} /$ week, fish intake once or more often in $2 \mathrm{~d}$, meat intake $\leq 2$ times/week, tsukemono intake once or more often per day, infrequent intake of soup with noodles, use of low-salt soya sauce and occasional drinking. The afore-mentioned cut-off values were determined based on the previous studies on the intake of eggs, fish and alcohol $^{(9,10,13-15)}$. For meat and tsukemono, a near median was used as the cut-off. Infrequent intake of soup with noodles and the use of low-salt soya sauce were used as markers of salt restriction. Because data on the amounts of alcohol consumed were not available, and the association between all-cause mortality and alcohol consumption is known to be J-shaped ${ }^{(15)}$, we chose occasional drinking as a component of a healthy reduced-salt Japanese diet. Moderate alcohol consumption was also a component of a Mediterranean $\operatorname{diet}^{(2)}$. If any single dietary component was part of a typical daily diet, it was scored as 1 and 0 otherwise. Thus, the total score ranged from 0 to 7 , with 0 being least healthy and 7 being most healthy. The participants were divided into approximate tertiles of dietary scores (0-2, 3 and 4-7 scores). To obtain trend $P$, the Mantel-Haenszel $\chi^{2}$ statistical test was used to detect deviation from linearity 
in the association between nominal variables and the categories according to the diet score, and the ANOVA was used to detect deviation from linearity in the association between continuous variables and the categories. To examine the association between the Reduced-Salt Japanese Diet Score and all-cause and cause-specific mortality, age -, sex- and multivariate-adjusted hazard ratios were calculated using a Cox proportional hazards model. For multivariate analyses, age, sex, BMI and cigarette smoking (never and past smokers, current smokers $<20$ cigarettes/d, current smokers 2040 cigarettes/d and current smokers $\geq 41$ cigarettes/d) were entered as covariates for model 1 . For model 2, hypertension and diabetes were added. The dietary score group 0-2 served as a reference for comparison with the other tertiles. Sensitivity analyses were performed on the afore-mentioned Cox analysis by excluding those who did not report a preferred food type, by stratifying the lower and higher age groups at median age, 49.3 years, and by stratifying by sex. To examine the association between each of the components of a ReducedSalt Japanese Diet Score and all-cause mortality, adjustments were made for the covariates in model 2 .

To estimate adjusted survival probabilities, we derived Kaplan-Meier survival curves after propensity score matching ${ }^{(16)}$. Variables used in the propensity score were selected from the non-dietary variables: age (years), men (\%), BMI $\left(\mathrm{kg} / \mathrm{m}^{2}\right)$, current smokers $(\%)$, systolic BP $(\mathrm{mmHg})$, diastolic BP $(\mathrm{mmHg})$, on hypertension drugs (\%), diabetes $(\%)$, serum total cholesterol $(\mathrm{mg} / \mathrm{l})$, albumin $(\mathrm{mg} / \mathrm{l})$, uric acid (mg/l) and creatinine (mg/l). After matching, adjusted survival curves were estimated separately for those participants who fell in the Japanese dietary grouping that ranged from 0 to 3 and for those in grouping strata 4 and higher. Comparison of the survival curves was based on the log-rank test. We further examined survival differences by the two groups according to the diet score, with age and sex as the dependent variables in a regression model. The statistical model used was a life table regression procedure, with a Weibull distribution assumption for failure time included. The variables used in the calculation of the propensity score were also compared by $t$ test and $\chi^{2}$ test to determine whether the propensity score matching was successful in mitigating risk factor differences.

\section{Results}

Baseline characteristics and all-cause mortality according to preferred food type

The baseline characteristics according to the preferred food type are shown in Table 1. In this table, we excluded 201 participants with missing data on a preferred food type. Relatively few participants preferred the Western food type. Participants in this group were younger, were more likely to be women and were less often hypertensive than participants who chose the other diet types. Those who preferred a Western type of diet ate meat more frequently and consumed fish and tsukemono less often than those in the other groups. The two markers of salt restriction (infrequent consumption of soup with noodles and the use of low-salt soya sauce) were more prevalent among those who preferred a Western diet. Small differences, but a significant trend in the Reduced-Salt Japanese Diet Score, were observed (trend $P<0 \cdot 0001$ ).

\section{Baseline characteristics according to Reduced-Salt Japanese Diet Score}

Table 2 shows the baseline characteristics according to tertiles of the Reduced-Salt Japanese Diet Score. As the score increased, the mean age and BMI increased, although the latter increase was modest. The proportion of women and the prevalence of hypertension, daily drinking and non-smoking also increased with diet score. The prevalence of diabetes and the mean serum total cholesterol concentration were not significantly different across the groups. As expected, the percentage with each component of the Reduced-Salt Japanese Diet Score increased as the score increased.

Table 1. Baseline characteristics according to preferred food type - National Integrated Project for Prospective Observation of Non-Communicable Diseases and its Trends in the Aged, 1980, 1980-99*

(Mean values and standard deviations)

\begin{tabular}{|c|c|c|c|c|c|c|c|}
\hline & \multicolumn{2}{|c|}{ Japanese } & \multicolumn{2}{|c|}{ Mixture } & \multicolumn{2}{|c|}{ Western } & \multirow[b]{2}{*}{ Trend $P$} \\
\hline & Mean & SD & Mean & SD & Mean & SD & \\
\hline Number at risk & \multicolumn{2}{|c|}{6505} & \multicolumn{2}{|c|}{1977} & \multicolumn{2}{|c|}{403} & \\
\hline Age (years) & $52 \cdot 2$ & $13 \cdot 0$ & $45 \cdot 9$ & $12 \cdot 4$ & $44 \cdot 7$ & $12 \cdot 2$ & $<0.0001$ \\
\hline Men (\%) & \multicolumn{2}{|c|}{48.4} & \multicolumn{2}{|c|}{$33 \cdot 7$} & \multicolumn{2}{|c|}{32.5} & $<0.0001$ \\
\hline $\mathrm{BMI}\left(\mathrm{kg} / \mathrm{m}^{2}\right)$ & $22 \cdot 8$ & $3 \cdot 2$ & $22 \cdot 5$ & 3.0 & $22 \cdot 4$ & $3 \cdot 1$ & 0.003 \\
\hline Hypertension (\%) & \multicolumn{2}{|c|}{$47 \cdot 8$} & \multicolumn{2}{|c|}{$36 \cdot 2$} & \multicolumn{2}{|c|}{31.8} & $<0.0001$ \\
\hline Diabetes (\%) & \multicolumn{2}{|c|}{$5 \cdot 8$} & \multicolumn{2}{|c|}{$3 \cdot 6$} & \multicolumn{2}{|c|}{4.5} & 0.0002 \\
\hline Daily drinkers (\%) & \multirow{2}{*}{\multicolumn{2}{|c|}{$\begin{array}{l}20.6 \\
35.8\end{array}$}} & \multicolumn{2}{|c|}{$23 \cdot 2$} & \multicolumn{2}{|c|}{$24 \cdot 3$} & $<0.0001$ \\
\hline Current smokers (\%) & & & \multicolumn{2}{|c|}{$25 \cdot 7$} & \multicolumn{2}{|c|}{$23 \cdot 1$} & $<0.0001$ \\
\hline Egg (/week) & 4.0 & $2 \cdot 8$ & $4 \cdot 0$ & $2 \cdot 7$ & 4.0 & $2 \cdot 7$ & 0.42 \\
\hline Fish (times/week) & $4 \cdot 8$ & 3.4 & 4.3 & 2.9 & 4.3 & 3.0 & $<0.0001$ \\
\hline Meat (times/week) & 3.5 & $2 \cdot 7$ & $4 \cdot 3$ & 3.0 & 4.7 & $3 \cdot 3$ & $<0.0001$ \\
\hline Tsukemono (times/week) & $9 \cdot 6$ & $5 \cdot 0$ & $8 \cdot 3$ & $5 \cdot 2$ & $6 \cdot 6$ & $5 \cdot 0$ & $<0.0001$ \\
\hline Infrequent consumption of soup with noodles (\%) & \multicolumn{2}{|c|}{$51 \cdot 0$} & \multicolumn{2}{|c|}{$55 \cdot 2$} & \multicolumn{2}{|c|}{62.5} & $<0.0001$ \\
\hline Use of low-salt soya sauce (\%) & \multicolumn{2}{|c|}{$16 \cdot 7$} & \multicolumn{2}{|c|}{$17 \cdot 3$} & \multicolumn{2}{|c|}{$18 \cdot 4$} & $<0.0001$ \\
\hline Reduced-Salt Japanese Diet Score & $3 \cdot 2$ & 1.1 & 3.0 & 1.1 & $3 \cdot 0$ & 1.1 & $<0.0001$ \\
\hline
\end{tabular}

*We excluded 201 participants in this table, who did not choose their preferred food type. To obtain trend PS, the Mantel-Haenszel $\chi^{2}$ statistical test was used for nominal variables, and the ANOVA for continuous variables. 
Table 2. Baseline characteristics according to tertiles of the Reduced-Salt Japanese Diet Score among 4018 men and 5068 women - National Integrated Project for Prospective Observation of Non-Communicable Diseases and its Trends in the Aged, 1980, 1980-99*

(Mean values and standard deviations)

\begin{tabular}{|c|c|c|c|c|c|c|c|}
\hline & \multicolumn{2}{|c|}{ Score $0-2$} & \multicolumn{2}{|c|}{ Score 3} & \multicolumn{2}{|c|}{ Score 4-7 } & \multirow[b]{2}{*}{ Trend $P$} \\
\hline & Mean & SD & Mean & SD & Mean & SD & \\
\hline No. at risk (total $=9086$ ) & \multicolumn{2}{|c|}{2719} & \multicolumn{2}{|c|}{3113} & \multicolumn{2}{|c|}{3254} & \\
\hline Age (years) & $49 \cdot 1$ & 13.5 & $50 \cdot 7$ & $13 \cdot 1$ & $51 \cdot 7$ & $13 \cdot 0$ & $<0.0001$ \\
\hline Men (\%) & \multicolumn{2}{|c|}{$49 \cdot 3$} & \multicolumn{2}{|c|}{43.6} & \multicolumn{2}{|c|}{40.5} & $<0.0001$ \\
\hline $\operatorname{BMI}\left(\mathrm{kg} / \mathrm{m}^{2}\right)$ & $22 \cdot 6$ & $3 \cdot 0$ & $22 \cdot 7$ & $3 \cdot 2$ & $22 \cdot 8$ & $3 \cdot 2$ & 0.003 \\
\hline Hypertension (\%) & \multicolumn{2}{|c|}{41.9} & \multicolumn{2}{|c|}{45.0} & \multicolumn{2}{|c|}{$47 \cdot 0$} & $<0.0001$ \\
\hline Diabetes (\%) & \multicolumn{2}{|c|}{$4 \cdot 6$} & \multirow{2}{*}{\multicolumn{2}{|c|}{$\begin{array}{c}5.6 \\
18.8\end{array}$}} & \multicolumn{2}{|c|}{$5 \cdot 4$} & 0.18 \\
\hline Daily drinkers (\%) & \multirow{2}{*}{\multicolumn{2}{|c|}{$\begin{array}{c}6.3 \\
35.4\end{array}$}} & & & \multirow{2}{*}{\multicolumn{2}{|c|}{$\begin{array}{l}36.1 \\
31.5\end{array}$}} & $<0.0001$ \\
\hline Current smokers (\%) & & & \multicolumn{2}{|c|}{$32 \cdot 2$} & & & $<0.0001$ \\
\hline $\mathrm{TCH}(\mathrm{mg} / \mathrm{l})$ & 1890 & 330 & 1890 & 340 & 1880 & 340 & 0.33 \\
\hline Egg $\leq 2$ eggs/week (\%) & \multicolumn{2}{|c|}{$10 \cdot 1$} & \multicolumn{2}{|c|}{29.5} & \multicolumn{2}{|c|}{$60 \cdot 3$} & $<0.0001$ \\
\hline Fish once or more often in $2 \mathrm{~d}(\%)$ & \multicolumn{2}{|c|}{$26 \cdot 2$} & \multicolumn{2}{|c|}{$35 \cdot 0$} & \multicolumn{2}{|c|}{$38 \cdot 8$} & $<0.0001$ \\
\hline Meat $\leq 2$ times/week (\%) & \multirow{2}{*}{\multicolumn{2}{|c|}{$\begin{array}{l}12 \cdot 7 \\
22 \cdot 0\end{array}$}} & \multicolumn{2}{|c|}{30.5} & \multicolumn{2}{|c|}{$56 \cdot 8$} & $<0.0001$ \\
\hline Tsukemono once or more often per day (\%) & & & \multirow{2}{*}{\multicolumn{2}{|c|}{$\begin{array}{l}35.6 \\
34.4\end{array}$}} & \multirow{2}{*}{\multicolumn{2}{|c|}{$\begin{array}{l}42 \cdot 4 \\
53 \cdot 3\end{array}$}} & $<0.0001$ \\
\hline Infrequent consumption of soup with noodles (\%) & \multicolumn{2}{|c|}{$12 \cdot 3$} & & & & & $<0.0001$ \\
\hline Use of low-salt soya sauce (\%) & \multicolumn{2}{|c|}{6.5} & \multicolumn{2}{|c|}{$23 \cdot 0$} & \multicolumn{2}{|c|}{70.6} & $<0.0001$ \\
\hline Occasional drinking (\%) & \multicolumn{2}{|c|}{8.8} & \multicolumn{2}{|c|}{30.4} & & & $<0.0001$ \\
\hline
\end{tabular}

No., number; $\mathrm{TCH}$, serum total cholesterol concentration.

*We defined a healthy Japanese diet based on seven components: egg intake $\leq 2$ eggs/week, fish intake once or more often in $2 \mathrm{~d}$, meat intake $\leq 2$ times/week, tsukemono (preserved roots or leaves of seasonal vegetables) intake once or more often per day, infrequent intake of soup with noodles, use of low-salt soya sauce and occasional drinking. If a dietary component was part of a typical daily diet, it was scored as 1 and 0 otherwise. Thus, the total Reduced-Salt Japanese Diet Score ranged from 0 to 7 , with 0 being least healthy and 7 being most healthy. To obtain trend Ps, the Mantel-Haenszel $\chi^{2}$ statistical test was used for nominal variables, and the ANOVA for continuous variables.

\section{All-cause and cause-specific mortality according to Reduced-} Salt Japanese Diet Score

During the 19 years of follow-up, there were 1823 deaths. In this group, 654 were from CVD, 299 from stroke, 131 acute myocardial infarction, 551 cancer and 119 non-cardiovascular, non-cancer inflammatory diseases ${ }^{(17)}$. Table 3 shows the total person-years, numbers of cases, hazard ratios and $95 \% \mathrm{CI}$ for all-cause and cause-specific mortality for each category of Reduced-Salt Japanese Diet Score after adjustment for age, sex and other risk factors (multivariate models 1 and 2). As the score increased, risk of death from all-cause mortality, CVD and stroke declined significantly in all models. Mortality from acute myocardial infarction, cancer and inflammatory diseases tended to decrease, but without statistical significance, a possible consequence of the relatively small number of such events. Similar results were observed after excluding participants with missing data on dietary preference. At high-age strata and in men, similar results were observed for all-cause mortality, CVD and stroke mortality. However, at low-age strata and in women, results were similar for all-cause mortality only. Significant differences by the groups according to the diet score were lost at low-age strata and in women for CVD and stroke mortality, probably because of the relatively smaller number of such events at low-age strata.

\section{Components of Reduced-Salt Japanese Diet Score and all- cause mortality}

The percentage of total participants who observed a healthy component of the Reduced-Salt Japanese Diet Score and the association of each component with all-cause mortality are shown in Table 4. The percentage of male participants who observed a healthy reduced-salt Japanese dietary component is also provided. Adherence to each of the healthy dietary components tended to be associated with lower mortality. Risk of death, however, was significantly lower for participants who ate tsukemono once or more often per day, consumed soup with noodles infrequently and drank alcohol occasionally.

\section{Kaplan-Meier survival estimates after propensity score matching}

The results from the propensity score matching are shown in Table 5. Fifty-eight participants with the Reduced-Salt Japanese Diet Score 4-7 were unmatched due to missing data ( $n$ 56) or failure to match on propensity scores $(n 2)$. As can be seen, significant differences in the average propensity score and the variables used in its calculation before matching in the two groups disappeared after matching. By contrast, a significant difference between the matched survival curves remained as shown in Fig. $1(P=0.0003$ by log-rank test). Survival differences by the group were significant when examined further using a regression model with a Weibull distribution that included adjustment for age and sex as the dependent variables (estimate $=-0.13$ (the lower score group compared with the higher score group), $P<0 \cdot 0001)$.

\section{Discussion}

The cut-off values for the egg, fish and drinking components were determined based on the previous studies ${ }^{(11,12,18-23)}$ Near-median cut-off values were used for meat and tsukemono. The low intake of meat is one of the characteristic features of the traditional Japanese diet and serves as a marker of reduced intake of SFA in the Japanese ${ }^{(3,4,18,19)}$. Although frequent intake of tsukemono is also a character- 
Table 3. All-cause and cause-specific mortality according to Reduced-Salt Japanese Diet Score among 9089 men and women - National Integrated Project for Prospective Observation of Non-Communicable Diseases and its Trends in the Aged, 1980, 1980-99*

(Hazard ratios (HR) and $95 \%$ confidence intervals)

\begin{tabular}{|c|c|c|c|c|c|c|}
\hline & \multirow{2}{*}{ Score $0-2$} & \multicolumn{2}{|r|}{ Score 3} & \multicolumn{2}{|c|}{ Score 4-7 } & \multirow[b]{2}{*}{ Trend $P$} \\
\hline & & $\mathrm{HR}$ & $95 \% \mathrm{Cl}$ & $\mathrm{HR}$ & $95 \% \mathrm{Cl}$ & \\
\hline Person-years & 46790 & \multicolumn{2}{|r|}{53772} & \multicolumn{2}{|c|}{56495} & \\
\hline All-cause death (total $=1823$ ) & 556 & \multicolumn{2}{|r|}{634} & \multicolumn{2}{|c|}{633} & \\
\hline Age-, sex-adjusted HR & 1 & 0.92 & $0.83,1.04$ & 0.78 & $0.70,0.88$ & $<0.0001$ \\
\hline \multicolumn{7}{|l|}{ Multivariate HR } \\
\hline Model 1 & 1 & 0.93 & $0.83,1.04$ & 0.78 & $0.69,0.87$ & $<0.0001$ \\
\hline Model 2 & 1 & 0.92 & $0.83,1.04$ & 0.78 & $0.70,0.88$ & $<0.0001$ \\
\hline CVD death (subtotal $=654$ ) & 200 & \multicolumn{2}{|r|}{220} & \multicolumn{2}{|c|}{234} & \\
\hline Age-, sex-adjusted HR & 1 & 0.90 & $0.75,1.09$ & 0.80 & $0.66,0.96$ & 0.017 \\
\hline \multicolumn{7}{|l|}{ Multivariate HR } \\
\hline Model 1 & 1 & 0.91 & $0.75,1.10$ & 0.79 & $0.65,0.95$ & 0.014 \\
\hline Model 2 & 1 & 0.91 & $0.75,1 \cdot 10$ & 0.80 & $0.66,0.97$ & 0.022 \\
\hline Stroke death $($ subtotal $=299)$ & 92 & \multicolumn{2}{|r|}{107} & \multicolumn{2}{|c|}{100} & \\
\hline Age-, sex-adjusted HR & 1 & 0.95 & $0.72,1.26$ & 0.74 & $0.56,0.99$ & 0.035 \\
\hline \multicolumn{7}{|l|}{ Multivariate HR } \\
\hline Model 1 & 1 & 0.96 & $0.73,1.27$ & 0.74 & $0.56,0.98$ & 0.031 \\
\hline Model 2 & 1 & 0.96 & $0.72,1.27$ & 0.75 & $0.56,0.99$ & 0.038 \\
\hline AMI death (subtotal = 131) & 40 & \multicolumn{2}{|r|}{42} & \multicolumn{2}{|c|}{49} & \\
\hline Age-, sex-adjusted HR & 1 & 0.83 & $0.55,1.26$ & 0.85 & $0.55,1.31$ & 0.39 \\
\hline \multicolumn{7}{|l|}{ Multivariate HR } \\
\hline Model 1 & 1 & 0.86 & $0.56,1.33$ & 0.82 & $0.54,1.25$ & 0.37 \\
\hline Model 2 & 1 & 0.85 & $0.55,1.32$ & 0.84 & $0.55,1.27$ & 0.42 \\
\hline Cancer death (subtotal $=551$ ) & 166 & \multicolumn{2}{|r|}{190} & \multicolumn{2}{|c|}{195} & \\
\hline Age-, sex-adjusted HR & 1 & 0.94 & $0.77,1.16$ & 0.86 & $0.70,1.05$ & 0.14 \\
\hline \multicolumn{7}{|l|}{ Multivariate HR } \\
\hline Model 1 & 1 & 0.95 & $0.77,1.17$ & 0.85 & $0.69,1.05$ & 0.12 \\
\hline Model 2 & 1 & 0.95 & $0.77,1.17$ & 0.85 & $0.69,1.05$ & 0.13 \\
\hline Non-CVD, non-cancer, Inflam. death (subtotal $=119$ ) & 40 & \multicolumn{2}{|r|}{37} & \multicolumn{2}{|c|}{42} & \\
\hline Age-, sex-adjusted HR & 1 & 0.81 & $0.52,1 \cdot 27$ & 0.74 & $0.48,1.14$ & 0.18 \\
\hline \multicolumn{7}{|l|}{ Multivariate HR } \\
\hline Model 1 & 1 & 0.80 & $0.51,1.25$ & 0.74 & $0.48,1.13$ & 0.17 \\
\hline Model 2 & 1 & 0.80 & $0.51,1.25$ & 0.74 & $0.48,1.14$ & 0.18 \\
\hline
\end{tabular}

AMI, acute myocardial infarction; Inflam., inflammatory disease.

*Multivariate, multivariate-adjusted Cox analysis. Model 1: adjusted for age, sex, BMl and smoking (never and ex-smokers, current smokers <20 cigarettes/d, current smokers $20-40$ cigarettes/d and current smokers $\geq 41$ cigarettes/d). Model 2: adjusted for model 1 covariates plus hypertension and diabetes.

istic feature of the traditional Japanese diet, it was unexpected to find that consuming tsukemono at least once a day was associated with a statistically significant lower risk of all-cause mortality. Many types of Japanese tsukemono are prepared in a traditional Japanese fashion with high reliance on salt. It may be, however, that the more healthy nutrient content of tsukemono outweighs the adverse consequences from consuming tsukemono with high sodium content. Conversely, the healthy nutritional value from eating unsalted tsukemono may be modest and offer little prognostic significance. Rather, its association with lower mortality may be through a high likelihood of being associated with a traditional Japanese diet. Those who eat tsukemono may consume meat less often and prefer foods that are commonly enjoyed with tsukemono, such as fish, vegetables, fruits and soya bean products.

Table 4. Components of Reduced-Salt Japanese Diet Score and all-cause mortality among 9089 men and women - National Integrated Project for Prospective Observation of Non-Communicable Diseases and its Trends in the Aged, 1980, 1980-99*

(Hazard ratios (HR) and $95 \%$ confidence intervals)

\begin{tabular}{|c|c|c|c|c|c|}
\hline Component & Total (\%) & Men \% & $\mathrm{HR}$ & $95 \% \mathrm{Cl}$ & $P$ \\
\hline Egg $\leq 2$ eggs/week & $36 \cdot 1$ & $40 \cdot 0$ & 0.93 & $0.84,1.02$ & $0 \cdot 11$ \\
\hline Fish once or more often in $2 d$ & 71.9 & $45 \cdot 9$ & 0.98 & $0.88,1.08$ & 0.67 \\
\hline Meat $\leq 2$ times/week & $38 \cdot 1$ & $40 \cdot 6$ & 0.97 & $0.88,1.06$ & 0.51 \\
\hline Tsukemono once or more often per day & $77 \cdot 0$ & 43.8 & 0.89 & $0.80,0.998$ & 0.045 \\
\hline Infrequent consumption of soup with noodles & $51 \cdot 3$ & $36 \cdot 3$ & 0.88 & $0.80,0.97$ & 0.007 \\
\hline Use of low-salt soya sauce & $16 \cdot 6$ & 41.8 & 0.99 & $0.88,1.12$ & 0.86 \\
\hline Occasional drinking & $21 \cdot 2$ & 55.4 & 0.81 & $0.71,0.92$ & 0.001 \\
\hline
\end{tabular}

Total (\%), percentage of total participants who had each component of Reduced-Salt Japanese Diet Score; men \%, percentage of men who had each component.

*Multivariate, multivariate-adjusted Cox analysis adjusted for age, sex, BMI, hypertension, diabetes and smoking (never and ex-smokers, current smokers <20 cigarettes/d, current smokers $20-40$ cigarettes/d and $>40$ cigarettes/d). 
Table 5. Variables used for propensity score matching and survival rate - National Integrated Project for Prospective Observation of Non-Communicable Diseases and its Trends in the Aged, 1980, 1980-99

(Mean values and standard deviations)

\begin{tabular}{|c|c|c|c|c|c|c|c|c|c|c|}
\hline & \multicolumn{4}{|c|}{ Before matching } & \multirow[b]{3}{*}{$P$} & \multicolumn{4}{|c|}{ After matching } & \multirow[b]{3}{*}{$P$} \\
\hline & \multicolumn{2}{|c|}{ Score 4-7 } & \multicolumn{2}{|c|}{ Score 0-3 } & & \multicolumn{2}{|c|}{ Score 4-7 } & \multicolumn{2}{|c|}{ Score $0-3$} & \\
\hline & Mean & SD & Mean & SD & & Mean & SD & Mean & SD & \\
\hline$n$ & \multicolumn{2}{|c|}{3254} & \multicolumn{2}{|c|}{5832} & & \multicolumn{2}{|c|}{3196} & \multicolumn{2}{|c|}{3196} & \\
\hline Age (years) & $51 \cdot 7$ & $13 \cdot 0$ & $50 \cdot 0$ & $13 \cdot 3$ & $<0.0001$ & $51 \cdot 6$ & $13 \cdot 0$ & $51 \cdot 6$ & 13.5 & 0.96 \\
\hline Men (\%) & \multicolumn{2}{|c|}{40.5} & \multicolumn{2}{|c|}{$46 \cdot 3$} & $<0.0001$ & \multicolumn{2}{|c|}{40.0} & \multicolumn{2}{|c|}{40.4} & 0.78 \\
\hline BMI $\left(\mathrm{kg} / \mathrm{m}^{2}\right)$ & $22 \cdot 8$ & $3 \cdot 2$ & $22 \cdot 6$ & $3 \cdot 1$ & 0.003 & $22 \cdot 8$ & 3.2 & $22 \cdot 8$ & $3 \cdot 2$ & 0.96 \\
\hline Current smokers (\%) & \multicolumn{2}{|c|}{31.5} & \multicolumn{2}{|c|}{$33 \cdot 7$} & 0.008 & \multicolumn{2}{|c|}{31.4} & \multicolumn{2}{|c|}{30.0} & 0.48 \\
\hline Systolic BP (mmHg) & $137 \cdot 0$ & $21 \cdot 2$ & $135 \cdot 2$ & $21 \cdot 1$ & $<0.0001$ & $137 \cdot 0$ & 21.2 & $136 \cdot 8$ & 21.9 & 0.68 \\
\hline Diastolic BP (mmHg) & 81.8 & $12 \cdot 2$ & $81 \cdot 0$ & $12 \cdot 1$ & 0.003 & 81.8 & $12 \cdot 2$ & $81 \cdot 3$ & $12 \cdot 4$ & 0.11 \\
\hline Hypertension drugs (\%) & \multicolumn{2}{|c|}{$10 \cdot 0$} & \multicolumn{2}{|c|}{$6 \cdot 9$} & $<0.0001$ & \multicolumn{2}{|c|}{$10 \cdot 0$} & \multicolumn{2}{|c|}{9.5} & 0.58 \\
\hline Diabetes (\%) & \multicolumn{2}{|c|}{5.4} & \multicolumn{2}{|c|}{$5 \cdot 1$} & 0.54 & \multicolumn{2}{|c|}{5.4} & \multicolumn{2}{|c|}{$5 \cdot 2$} & 0.78 \\
\hline $\mathrm{TCH}(\mathrm{mg} / \mathrm{l})$ & 1880 & 340 & 1890 & 340 & 0.24 & 1880 & 340 & 1870 & 330 & 0.47 \\
\hline Albumin (mg/l) & 44 & 03 & 44 & 03 & 0.66 & 44 & 03 & 44 & 03 & 0.91 \\
\hline Uric acid (mg/l) & 49 & 13 & 50 & 13 & 0.07 & 49 & 13 & 50 & 13 & 0.76 \\
\hline Creatinine (mg/l) & 9.3 & 1.7 & 9.4 & $2 \cdot 0$ & 0.02 & $9 \cdot 3$ & 1.7 & $9 \cdot 3$ & $2 \cdot 1$ & 0.52 \\
\hline Propensity score & 0.64 & 0.05 & 0.65 & 0.05 & $<0.0001$ & 0.64 & 0.05 & 0.63 & 0.05 & 0.84 \\
\hline (min, $\max )$ & 0.44 & 0.80 & 0.12 & 0.82 & & 0.44 & 0.80 & 0.42 & 0.76 & \\
\hline
\end{tabular}

$\mathrm{BP}$, blood pressure; $\mathrm{TCH}$, serum total cholesterol concentration.

We merely do not eat foods, but in certain patterns ${ }^{(20)}$, such as those in the Mediterranean and Japanese dietary patterns. Because of highly interrelated dietary exposures, dietary patterns, rather than the specific effects of nutrients or foods, have gained increasing attention ${ }^{(1,2)}$. Although one drawback of the traditional Japanese diet is a high intake of salt, reduction in salt intake by the Japanese for the last three decades has been considered as one of the chief explanations for the decline in not only stroke but also stomach cancer mortality in $\operatorname{Japan}^{(5,6,21,23)}$. This is consistent with the finding in the present report that infrequent consumption of soups with noodles, a marker of low salt intake, was associated with a significantly lower risk of all-cause mortality by itself.

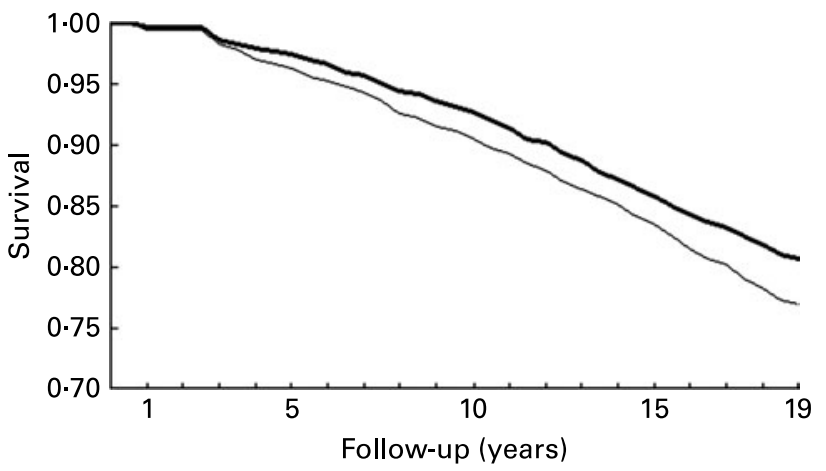

Fig. 1. Kaplan-Meier survival curve after propensity score matching. Significant differences in the average propensity score and the variables used in its calculation before matching in the two groups disappeared after matching. By contrast, a significant difference between the matched survival curves remained as shown in the figure $(P=0.0003$ by log-rank test). Survival differences by the group were significant when examined further using a regression model with a Weibull distribution that included adjustment for age and sex as the dependent variables $(P<0.0001)$. The thick line indicates survival for the participants with the Reduced-Salt Japanese Diet Score 4-7 and the thin line with the Reduced-Salt Japanese Diet Score 0-3.

\section{Strengths and limitations of the study}

The strengths of the present study include its prospective design and the follow-up of a randomly selected sample from the general population of Japan with a high response rate $(76 \%)$. Since the study includes both men and women with a broad range of ages, findings are likely to be generalisable to middle-aged and elderly Japanese men and women.

As in any long-term follow-up study, however, there are several weaknesses. First, we surveyed essential nutritional components by the food-frequency method once at the baseline. As a result, we have no data on total caloric intake or total dietary intake of cholesterol or saturated and PUFA. To obtain these data, detailed food records or 24-h recalls are needed. However, these methods are impractical and seldom used as the primary method for estimating usual intake in large-scale epidemiological studies. A second limitation is that the items used for the food-frequency method were not large in number, and has not been validated. We do not have data to what extent these foods contribute to the average energy intake of the studied participants. We also do not have frequency data on tofu, other soya bean products and vegetables and fruits. A high intake of these foods may also be characteristic features of the traditional Japanese diet. Several studies indicate that these foods have beneficial effects on some cause-specific mortality ${ }^{(24,25)}$. In addition, although the use of near-median values as cut-points for the consumption of meat and tsukemono appears arbitrary, they were chosen in accordance with their use in the previous studies of the Mediterranean $\operatorname{diet}^{(2)}$. Unfortunately, while the intake of tsukemono, infrequent consumption of soup with noodles and occasional drinking appeared to have the strongest association with a reduced risk of mortality, we cannot be certain that the other components of the Japanese diet are less important. As in any observational study, it is difficult to identify specific dietary effects due to multicollinearity that exists among food item intake. Within each component of the Japanese diet, there can also be considerable heterogeneity in nutrient 
content. In addition, overlap between components often occurs with the sharing of common ingredients or in how they are prepared and served. To better identify the effects of specific nutrients on mortality would require a controlled clinical trial. It may also be that dietary factors need to be considered in combination for an effect on longevity to be observed. An additional limitation is that we used mortality data as end points, which may lead to the misclassification of the cause of deaths. However, it has been reported that the death-certificate diagnosis for stroke and cancer in Japan is quite accurate $^{(26)}$. Possible misclassification of acute myocardial infarction as 'heart failure' is also not an issue in the present report since both outcomes are collectively categorised as $\operatorname{CVD}^{(27)}$.

\section{Conclusions}

Adherence to a healthy Japanese diet was associated with an approximate $20 \%$ lower rate of all-cause and cardiovascular mortality. While Japanese are exceptionally long lived, placing greater emphasis on the intake of foods that are low in salt could increase longevity in Japan further.

\section{Acknowledgements}

The authors thank all members of the Japanese Association of Public Health Center Directors and all staffs of the public health centres who cooperated with the present study. Investigators and members of the research group are listed in the appendix of Nakamura et al. ${ }^{(11)}$. Y. N. participated in designing and conducting the study, analysing and interpreting the data, and writing and preparing the manuscript. H. U. was the principal investigator and participated in designing and conducting the study and analysing and interpreting the data. T. O., R. D. A. and A. O. participated in conducting the study and analysing and interpreting the data. T. K. and Y. K. participated in managing and interpreting the data. T. H. participated in managing the data and conducting the study. We all have no conflicts of interest to disclose. The present study was supported by the Research Grant for Cardiovascular Diseases (7A-2) from the Ministry of Health, Labour and Welfare and a Health and Labour Sciences Research Grant, Japan (Comprehensive Research on Aging and Health: H11-Chouju-046, H14-Chouju-003, H17Chouju-012), and by the Japan Society for the Promotion of Science Invitation Fellowship Programmes for Research, Japan.

\section{References}

1. Huijbregts P, Feskens E, Rasanen L, et al. (1997) Dietary pattern and 20 year mortality in elderly men in Finland, Italy, and The Netherlands: longitudinal cohort study. BMJ 315, $13-17$.

2. Trichopoulou A, Costacou T, Bamia C, et al. (2003) Adherence to a Mediterranean diet and survival in a Greek population. $N$ Engl J Med 348, 2599-2608.

3. Robertson TL, Kato H, Gordon T, et al. (1977) Epidemiologic studies of coronary heart disease and stroke in Japanese men living in Japan, Hawaii and California. Coronary heart disease risk factors in Japan and Hawaii. Am J Cardiol 39, 244-249.
4. Carroll KK (1975) Experimental evidence of dietary factors and hormone-dependent cancers. Cancer Res 35, 3374-3383.

5. Ueshima H, Zhang XH \& Choudhury SR (2000) Epidemiology of hypertension in China and Japan. J Hum Hypertens 14, $765-769$.

6. Kubo M, Kiyohara Y, Kato I, et al. (2003) Trends in the incidence, mortality, and survival rate of cardiovascular disease in a Japanese community: the Hisayama study. Stroke 34, 2349-2354.

7. Nagata C, Takatsuka N, Shimizu N, et al. (2004) Sodium intake and risk of death from stroke in Japanese men and women. Stroke 35, 1543-1547.

8. Okamura T, Kadowaki T, Hayakawa T, et al. (2003) What cause of mortality can we predict by cholesterol screening in the Japanese general population? J Intern Med 253, 169-180.

9. Nakamura Y, Okamura T, Tamaki S, et al. (2004) Egg consumption, serum cholesterol, and cause-specific and all-cause mortality: the national integrated project for prospective observation of non-communicable disease and its trends in the aged, 1980 (NIPPON DATA80). Am J Clin Nutr 80, 58-63.

10. Nakamura $Y$, Ueshima H, Okamura T, et al. (2005) Association between fish consumption and all-cause and cause-specific mortality in Japan: NIPPON DATA80, 1980-99. Am J Med 118, 239-245.

11. Nakamura M, Sato S \& Shimamoto T (2003) Improvement in Japanese clinical laboratory measurements of total cholesterol and HDL-cholesterol by the US cholesterol reference method laboratory network. J Atheroscler Thromb 10, 145-153.

12. Kromhout D, Bosschieter EB \& de Lezenne Coulander C (1985) The inverse relation between fish consumption and 20-year mortality from coronary heart disease. $N$ Engl J Med 312, $1205-1209$.

13. Daviglus ML, Stamler J, Orencia AJ, et al. (1997) Fish consumption and the 30-year risk of fatal myocardial infarction. $N$ Engl J Med 336, 1046-1053.

14. Iso H, Kobayashi M, Ishihara J, et al. (2006) Intake of fish and $n 3$ fatty acids and risk of coronary heart disease among Japanese: the Japan Public Health Center-based (JPHC) Study Cohort I. Circulation 113, 195-202.

15. Tsugane S, Fahey MT, Sasaki S, et al. (1999) Alcohol consumption and all-cause and cancer mortality among middle-aged Japanese men: seven-year follow-up of the JPHC study Cohort I. Japan Public Health Center. Am J Epidemiol 150, $1201-1207$.

16. Parsons LS (2001) Reducing bias in a propensity score matchedpair sample using greedy matching techniques. In Proceedings of the Twenty-sixth Annual SAS® Users Group International Conference, pp. 214-226. Cary, NC: SAS Institute Inc. www2.sas.com/proceedings/sugi26/p214-26.pdf

17. Jacobs DR Jr, Andersen LF \& Blomhoff R (2007) Whole-grain consumption is associated with a reduced risk of noncardiovascular, noncancer death attributed to inflammatory diseases in the Iowa Women's Health Study. Am J Clin Nutr 85, 1606-1614.

18. Ueshima H, Iida M, Shimamoto T, et al. (1982) Dietary intake and serum total cholesterol level: their relationship to different lifestyles in several Japanese populations. Circulation 66, 519-526.

19. Ueshima H, Okayama A, Saitoh S, et al. (2003) Differences in cardiovascular disease risk factors between Japanese in Japan and Japanese-Americans in Hawaii: the INTERLIPID Study. $J$ Hum Hypertens 17, 631-639.

20. Jacques PF \& Tucker KL (2001) Are dietary patterns useful for understanding the role of diet in chronic disease? Am J Clin Nutr 73, 1-2

21. Ueshima H, Tatara K \& Asakura S (1987) Declining mortality from ischemic heart disease and changes in coronary risk factors in Japan, 1956-1980. Am J Epidemiol 125, 62-72. 
22. Tokui N, Yoshimura T, Fujino Y, et al. (2005) Dietary habits and stomach cancer risk in the JACC Study. J Epidemiol 15, Suppl. 2, S98-S108.

23. Tsugane S, Sasazuki S, Kobayashi M, et al. (2004) Salt and salted food intake and subsequent risk of gastric cancer among middle-aged Japanese men and women. Br J Cancer 90, $128-134$.

24. Sauvaget C, Nagano J, Allen N, et al. (2003) Vegetable and fruit intake and stroke mortality in the Hiroshima/Nagasaki Life Span Study. Stroke 34, 2355-2360.
25. Yamamoto S, Sobue T, Kobayashi M, et al. (2003) Soy, isoflavones, and breast cancer risk in Japan. J Natl Cancer Inst $\mathbf{9 5}$, 906-913.

26. Ron E, Carter R, Jablon S, et al. (1994) Agreement between death certificate and autopsy diagnoses among atomic bomb survivors. Epidemiology 5, 48-56.

27. Saito I, Folsom AR, Aono H, et al. (2000) Comparison of fatal coronary heart disease occurrence based on population surveys in Japan and the USA. Int J Epidemiol 29, $837-844$. 


\section{Appendix NIPPON DATA80 Dietary Questionnaire}

Q1. Do you eat breakfast daily? Yes

Yes $\quad$ No

Q2. Do you daily eat green or yellow vegetables, such as carrot or spinach? $\quad$ Yes $\quad$ No

Q3. Do you daily eat fruits? $\quad$ Yes

Q4. Do you daily eat salad or fresh vegetables? $\quad$ Yes $\quad$ No

Q5. Do you daily eat meat, fish or egg? $\quad$ Yes $\quad$ No

Q6. Do you daily drink milk? $\quad$ Yes $\quad$ No

Q7. Do you eat soya bean products, such as natto (fermented soya beans) or tofu more $\quad$ Yes $\quad$ No than three times per week?

Q8. Do you eat foods cooked with oil more than once daily? $\quad$ Yes $\quad$ No

Q9. Do you eat seaweed, such as kombu or laver more than three times per week?

Q10. Do you eat potatoes more than three times per week?

For each food listed on Q11-Q16, please check the box indicating how often you eat, on average

Q11. Egg (how many)

$\geq 2 / \mathrm{d} \quad 1 / \mathrm{d} \quad 1 / 2 \mathrm{~d} \quad 1-2 /$ week $\quad<1 /$ week

Q12. Fish (how often)

Q13. Meat (including ham and sausage, how often)

Q14. Noodles (how often)

Q15. Tsukemono (how often)

Q16. Soup (including miso soup, how often)

Q17. Please select one food from the list that you like to eat the most:
(1) Beef
(2) Pork
(3) Poultry
(4) Undecidable

Q18. Please select one dish from the list that you like to eat the most:
(1) Egg food
(2) Meat dishes
(3) Fish dishes
(4) Tofu food
(5) A vegetable dish

Q19. Please select one food combination from the list that you like to eat the most:

(1) Rice bowl + sashimi + miso soup + tsukemono

(2) Bread + hamburger steak + potage soup + salad

(3) Rice bowl + hamburger steak + miso soup + salad

Q20. Which type of seasoning do you like best to eat with?
(1) Thick
(2) Intermediate
(3) Light

Q21. How do you eat tsukemono?
(1) As it is
(2) Seasoning with soya sauce
(3) Seasoning with sodium glutamate
(4) Seasoning with soya sauce plus sodium glutamate

\section{From Q22 to Q31, please choose one that fits best your recent eating habit}

Q22. Do you try to eat modest amount of food?

Q23. Do you often eat processed foods, such as ham, sausage, kamaboko or a tubular fish meat?

Q24. Are you not satisfied if you do not eat with tsukemono?

Q25. Are you not satisfied if you do not eat with a kind of soup?

Q26. Do you take soup infrequently with noodles? 
Q27. When you eat tofu served cold, how do you season it with soya sauce?

(1) Dip it in a small dish with soya sauce

(2) Pour soya sauce over tofu.

Q28. When you eat curry and rice, do you pour Worcestershire sauce or soya sauce over it? Yes No

Q29. Are you trying to eat soups less often?

Yes

No

Q30. Have you ever used low-salt soya sauce?

Yes

Yes No

Q31. Are you trying to eat tsukudani (a shellfish boiled in sweetened soya sauce), shiokara (fish guts pickled in salt), or salted salmon less often? 